\title{
Effect of Carbamazepine on the Single Oral Dose Pharmacokinetics of Alprazolam
}

\author{
Hanako Furukori, M.D., Koichi Otani, M.D., Ph.D., Norio Yasui, M.D., Ph.D., \\ Tsuyoshi Kondo, M.D., Ph.D., Sunao Kaneko, M.D., Ph.D., Ritsuko Shimoyama, B.S., \\ Tadashi Ohkubo, Ph.D., Takako Nagasaki, B.S., and Kazunobu Sugawara, Ph.D.
}

The effect of carbamazepine, an inducer of cytochrome $P 450$ (CYP) $3 A 4$, on the single oral dose pharmacokinetics of alprazolam was examined in a double-blind, randomized crossover study with two phases. Seven healthy male subjects took carbamazepine $300 \mathrm{mg} /$ day or matched placebo orally for 10 days, and on the 8th day they took a single oral $0.8 \mathrm{mg}$ dose of alprazolam. Blood samples were taken and psychomotor function was assessed by the Digit Symbol Substitution Test, Visual Analog Scale, and UKU Side Effect Rating Scale up to $48 \mathrm{~h}$ after alprazolam dosing. Carbamazepine significantly ( $\mathrm{p}<.01$ to .001) decreased the plasma alprazolam concentrations during the elimination phase. Carbamazepine significantly $(\mathrm{p}<.001)$ increased the apparent oral clearance ( $0.90 \pm 0.21 \mathrm{vs} .2 .13 \pm 0.54 \mathrm{ml} /$ $\mathrm{min} / \mathrm{kg})$ and shortened the elimination half-life $(17.1 \pm 4.9$ vs. $7.7 \pm 1.7 \mathrm{~h})$, with no significant effect on the peak plasma concentration $(11.7 \pm 1.5 \mathrm{vs} .13 .0 \pm 3.5 \mathrm{ng} / \mathrm{ml})$. The majority of psychomotor function parameters during the carbamazepine treatment were not significantly different from those during the placebo treatment, probably because of the sedative effect of carbamazepine itself. The present study suggests that carbamazepine decreases plasma concentration of alprazolam by inducing its metabolism. It also supports the previous studies, suggesting that alprazolam is metabolized predominantly by CYP3A4. [Neuropsychopharmacology 18: 364-369, 1998] (c) 1998 American College of Neuropsychopharmacology. Published by Elsevier Science Inc.
KEY WORDS: Alprazolam; Carbamazepine; Single oral dose; Pharmacokinetics; Metabolism; CYP3A4

The triazolobenzodiazepine alprazolam is used extensively in the treatment of anxiety and panic disorders (Greenblatt and Wright 1993). A significant concentration-effect relationship for alprazolam has been suggested in the treatment of panic disorder; optimal reduction of anxiety occurs in the plasma concentration range of 20-40 ng/ml, whereas the central nervous sys-

From the Department of Neuropsychiatry (HF, NY, TK, SK) and Pharmacy (RS, TO, TN, KS), Hirosaki University Hospital, Hirosaki, Japan; Department of Neuropsychiatry (KO), Yamagata University School of Medicine, Yamagata, Japan.

Address correspondence to: Tsuyoshi Kondo, M.D., Ph.D., Department of Neuropsychiatry, Hirosaki University Hospital, Hon-cho 53, Hirosaki 036, Japan.

Received May 5, 1997; accepted August 12, 1997. tem (CNS) depressant side effects increase progressively at higher plasma concentrations (Greenblatt and Wright 1993).

Alprazolam is metabolized primarily by the hepatic microsomal oxidation, yielding 4 - and $\alpha$-hydroxyalprazolam as its principal metabolites (Greenblatt and Wright 1993). Previous studies have suggested that neither cytochrome P450 (CYP) 2D6 (Bertilsson et al. 1988) nor CYP2C19 (Otani et al. 1997a) is involved in the metabolism of alprazolam. Meanwhile, the in vitro study by von Moltke et al. (1994) has shown that ketoconazole, an inhibitor of CYP3A4 (Olkkola et al. 1994; Watkins 1994), inhibits the 4 - and $\alpha$-hydroxylation of alprazolam, suggesting that alprazolam is metabolized by CYP3A4. Furthermore, Yasui et al. (1996) have reported that erythromycin, an inhibitor of CYP3A4 (Olkkola et al. 1993; Watkins 1994), inhibits the metabolism of al- 
prazolam in healthy volunteers, providing in vivo evidence for the involvement of CYP3A4.

Carbamazepine has been used increasingly in the treatment of psychiatric disorders (Siris 1993; Post et al. 1994; Otani et al. 1996a). However, it has been suggested that carbamazepine induces the metabolism of several psychotropic drugs (Arana et al. 1986; Backman et al. 1996; Otani et al. 1996b; 1997b) by a not fully characterized enzyme induction. Previous studies have suggested that carbamazepine induces CYP3A4 (Wietholtz et al. 1989; Pirmohamed et al. 1994; Yue et al. 1994), but not CYP1A2 (Wietholtz et al. 1989), CYP2D6 (Yue et al. 1994) nor UDP-glucuronosyltransferases (Yue et al. 1994). Therefore, carbamazepine may decrease plasma concentration of alprazolam by inducing its metabolism. In fact, Arana et al. (1988) has reported that carbamazepine decreased plasma concentration of alprazolam in one psychiatric patient. However, there has been no systematic study on the effect of carbamazepine on the plasma concentration and/or metabolism of alprazolam.

Therefore, we studied the effect of carbamazepine on the single oral dose pharmacokinetics of alprazolam in healthy volunteers. We also expected that the present study would provide further evidence for the involvement of CYP3A4 in the metabolism of alprazolam.

\section{METHODS}

\section{Subjects}

The subjects were seven healthy male volunteers. The mean \pm SD of age was $32.7 \pm 6.6$ years, and that of body weight was $60.9 \pm 4.6 \mathrm{~kg}$. Three subjects were smokers ( $\geq 10$ cigarettes/day), and the remaining four were nonsmokers. The study protocol was approved by the Ethics Committee of Hirosaki University Hospital, and each subject gave his written informed consent before the study.

\section{Protocol}

The study was conducted in a double-blind, randomized crossover manner, with at least a 6-week washout period. The subjects were allocated randomly to one of the two treatment sequences, placebo-carbamazepine or carbamazepine-placebo. Carbamazepine $100 \mathrm{mg}$ as the tablet formulation (Tegretol, Novartis Pharma, Tokyo, Japan) or matched placebo was given orally three times a day (at 7:00 A.M., 3:00 P.M. and 11:00 P.M.) for 10 days. After overnight fast at 9:00 A.M. of the 8th day, a single oral $0.8 \mathrm{mg}$ dose of alprazolam (Solanax, Pharmacia \& Upjohn, Tokyo, Japan) as the tablet formulation was given with a cup of tap water. No food was allowed until $3 \mathrm{~h}$ after alprazolam dosing. The blood samples (10 ml each) for alprazolam measurement were taken before and at $0.5,1,2,3,4,6,8,10,12,24,36$, and $48 \mathrm{~h}$ after alprazolam dosing. The blood samples for carbamazepine measurement were taken at 7:00 A.M. and 9:00 A.M. on the 8th day. At times of blood samplings, psychomotor function was assessed using the Digit Symbol Substitution Test (DSST) adapted from the Wechsler Adult Intelligence Scale in $3 \mathrm{~min}$, the Visual Analog Scale (VAS) of Mood and Subjective States (Greenblatt et al. 1977), and the item Sleepiness of the UKU Side Effect Rating Scale (Lingjaerde et al. 1987).

\section{Assay}

Plasma alprazolam concentrations were measured in duplicate by the high-performance liquid chromatography (HPLC) method of Nagasaki et al. (1997). The lowest limit of detection was $0.5 \mathrm{ng} / \mathrm{ml}$. Plasma carbamazepine concentrations were measured in duplicate by the following HPLC method recently developed in our laboratory. Briefly, to $1 \mathrm{ml}$ of plasma sample $90 \mathrm{ng}$ of 2-methylcarbamazepine was added as an internal standard. The extraction was conducted using a Sep-Pak $C_{18}$ cartridge (Waters Chromatography, Milford, MA). The HPLC system consisted of a Rheodyne Model 7125 injector (Rheodyne, Cotati, CA), a stainless-steel column $\left(150 \mathrm{~mm} \times 4.6 \mathrm{~mm}\right.$ ID) packed with Develosil $\mathrm{C}_{8}-5$ stationary phase ( $5 \mu \mathrm{m}$, Nomura Chemicals, Seto, Japan), a Philips, Pye Unicam PU 4010 pump, and a Pye Unicam PU 4020 UV detector (Pye Unicam, Cambridge, U.K.). The wavelength was set at $254 \mathrm{~nm}$. The mobile phase consisted of $0.5 \%$ ( $\mathrm{pH} 2.5$ ) monobasic potassium phosphate and acetonitrile $(67: 33, \mathrm{v} / \mathrm{v})$. The flow rate was $1.0 \mathrm{ml} / \mathrm{min}$ at ambient temperature. The detailed procedure will be reported elsewhere.

\section{Data Analysis}

The elimination rate constant $(\mathrm{k})$ of alprazolam was estimated from the linear regression analysis of the terminal log-linear concentration-time data, and the elimination half-life (t1/2) was calculated from $0.693 / \mathrm{k}$. During the placebo treatment, the area under the plasma concentration-time curve from 0 to $48 \mathrm{~h}$ [AUC $(0-48)$ ] was calculated by the trapezoidal rule. During the carbamazepine treatment the AUC(0-24) was calculated, because plasma alprazolam concentrations were detectable up to $24 \mathrm{~h}$ only. The AUC from $0 \mathrm{~h}$ to infinity [AUC $(0-\infty)$ ], or total AUC, was calculated from AUC $(0-$ 24) $+\mathrm{C}_{24} / \mathrm{k}$ or $\operatorname{AUC}(0-48)+\mathrm{C}_{48} / \mathrm{k}$, in which $\mathrm{C}_{24}$ and $\mathrm{C}_{48}$ are the plasma alprazolam concentrations at 24 and $48 \mathrm{~h}$, respectively. The apparent oral clearance (CLoral) and volume of distribution $(\mathrm{Vd})$ were estimated from CLoral $=$ dose $/$ total AUC and $\mathrm{Vd}=$ CLoral $/ \mathrm{k}$, respectively. The peak plasma concentration (Cmax) and the time to $\mathrm{Cmax}(\mathrm{tmax})$ were determined graphically. 


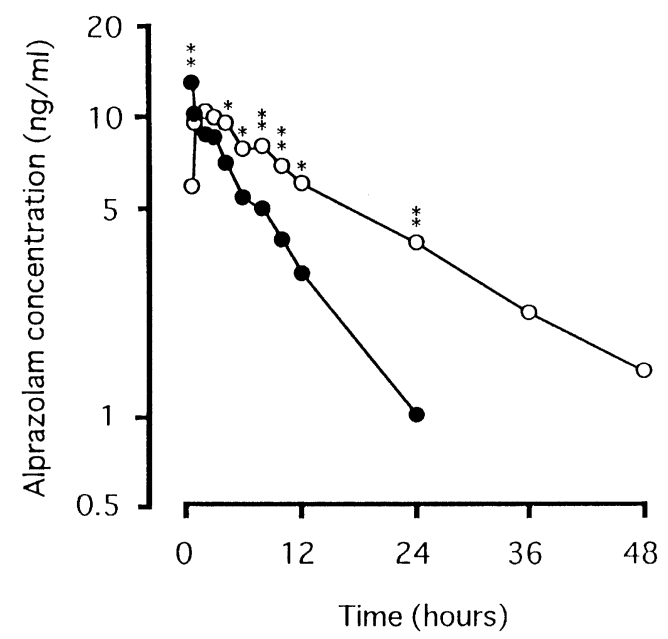

Figure 1. Mean plasma concentration-time data after a single oral $0.8 \mathrm{mg}$ dose of alprazolam during the treatment with placebo or carbamazepine. Open and solid circles indicate the mean data during the treatments with placebo and carbamazepine, respectively. Asterisks indicate significant differences between the two treatments: ${ }^{*} p<.01$; ${ }^{* *} p<.001$.

Statistical analysis was performed by a paired $t$-test. A $p$ value of less than .05 was considered statistically significant.

\section{RESULTS}

\section{Pharmacokinetic Assessment}

The mean plasma alprazolam concentration-time data during the treatment with placebo or carbamazepine are shown in Figure 1, and the mean $( \pm \mathrm{SD})$ pharmacokinetic parameters of alprazolam are summarized in Table 1.

In all subjects, plasma alprazolam concentrations were detectable up to $48 \mathrm{~h}$ during the placebo treatment, but they were detectable up to $24 \mathrm{~h}$ only during the carbamazepine treatment (Figure 1). Carbamazepine significantly ( $p<.01$ to .001$)$ decreased the mean plasma alprazolam concentrations at 4 to $24 \mathrm{~h}$ (Figure 1 ). The mean plasma alprazolam concentration at $0.5 \mathrm{~h}$ during the carbamazepine treatment was significantly $(p<$ .001) higher than that during the placebo treatment (Figure 1$)$. Carbamazepine significantly $(p<.001)$ increased the mean CLoral and shortened the mean elimination $t 1 / 2$ of alprazolam compared with placebo (Table 1). In all subjects, carbamazepine increased the CLoral and shortened the elimination $\mathrm{t} 1 / 2$. Carbamazepine did not significantly change the mean Cmax, tmax nor $\mathrm{Vd}$ (Table 1).

The mean $( \pm \mathrm{SD})$ plasma carbamazepine concentrations at 7:00 A.M. and 9:00 A.M. were 4.2 \pm 1.1 and $5.8 \pm$ $1.0 \mu \mathrm{g} / \mathrm{ml}$, respectively.

\section{Pharmacodynamic Assessment}

The mean psychomotor function parameters assessed by the DSST, VAS, and the item Sleepiness of the UKU Scale during the treatment with placebo or carbamazepine are shown in Figure 2. There were no significant differences in the mean DSST and UKU Scale scores between the two treatments at any time points. Although thinking speed was significantly $(p<.05)$ slower at $1 \mathrm{~h}$ and faster at $36 \mathrm{~h}$ during the carbamazepine treatment than during the placebo treatment, no significant differences were found in other items of the VAS between the two treatments at any time points.

\section{DISCUSSION}

In the present study, carbamazepine markedly decreased plasma alprazolam concentrations during the elimination phase. Furthermore, carbamazepine markedly increased the CLoral and shortened the elimination $\mathrm{t} 1 / 2$ of alprazolam. These results indicate that carbamazepine, an inducer of CYP3A4 (Wietholtz et al. 1989; Pirmohamed et al. 1994), induces the metabolism of al-

Table 1. Pharmacokinetic Parameters of Alprazolam after a Single Oral $0.8 \mathrm{mg}$ Dose during the Treatment with Placebo or Carbamazepine

\begin{tabular}{lccc}
\hline Parameters & Placebo & Carbamazepine & Significance \\
\hline Cmax $(\mathrm{ng} / \mathrm{ml})$ & $11.7 \pm 1.5$ & $13.0 \pm 3.5$ & $\mathrm{NS}$ \\
$\operatorname{tmax}(\mathrm{h})$ & $1.6 \pm 0.7$ & $1.0 \pm 0.9$ & $\mathrm{NS}$ \\
CLoral $(\mathrm{ml} / \mathrm{min} / \mathrm{kg})$ & $0.90 \pm 0.21$ & $2.13 \pm 0.54$ & $p<.001$ \\
Vd $(\mathrm{L} / \mathrm{kg})$ & $1.30 \pm 0.43$ & $1.36 \pm 0.24$ & $\mathrm{NS}$ \\
Elimination t1/2 (h) & $17.1 \pm 4.9$ & $7.7 \pm 1.7$ & $p<.001$ \\
\hline
\end{tabular}

The values on the table are means \pm SD.

Abbreviations: $\mathrm{Cmax}=$ peak plasma concentration; tmax $=$ time to $\mathrm{Cmax} ; \mathrm{CLoral}=$ apparent oral clearance; $\mathrm{Vd}=$ apparent volume of distribution; $\mathrm{t} 1 / 2=$ half-life. 
A. DSST

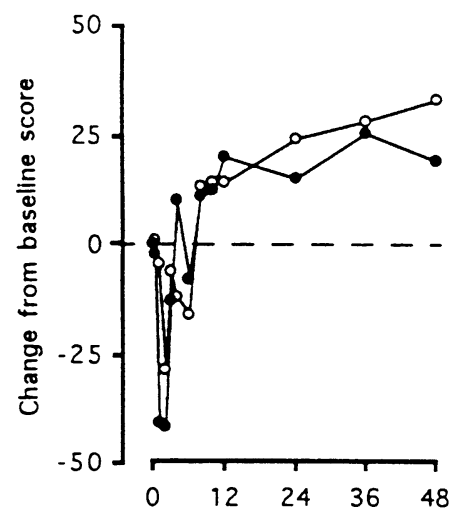

B. VAS

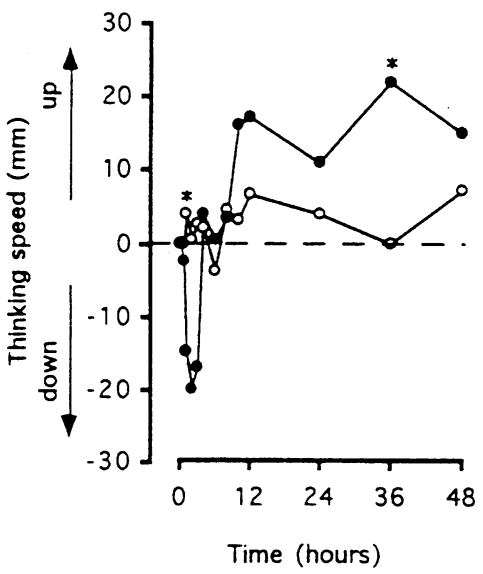

C. UKU Scale

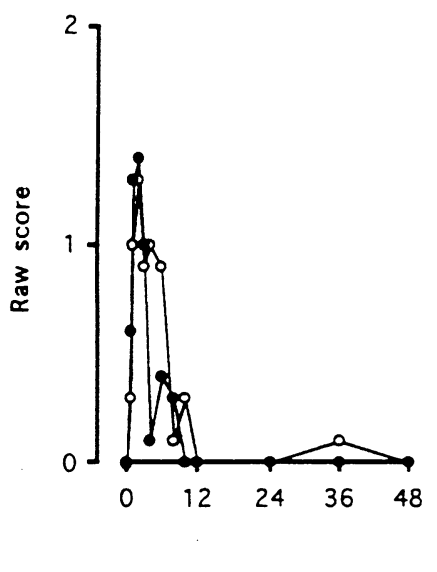

Figure 2. Mean psychomotor function parameters after a single oral $0.8 \mathrm{mg}$ dose of alprazolam during the treatment with placebo or carbamazepine. Open and solid circles indicate the mean data during the treatments with placebo and carbamazepine, respectively. Scores on the Digit Symbol Substitution Test (DSST), Visual Analog Scale (VAS) (only the item Thinking Speed for brevity), and UKU Side Effect Rating Scale (the item Sleepiness) are shown in A, B, and C, respectively. For the DSST and VAS, each time point is the mean increase or decrease over the predose score at the corresponding postdose times, and for the UKU Scale it is the mean raw score. Asterisks indicate significant differences between the two treatments: ${ }^{*} p<.05$.

prazolam. Therefore, the present study clearly indicates the involvement of CYP3A4 in the metabolism of alprazolam, supporting the results of previous in vitro (von Moltke et al. 1994) and in vivo (Yasui et al. 1996) studies.

Backman et al. (1996) have compared the single oral dose pharmacokinetics of midazolam, which is a probe drug for the CYP3A4 activity (Watkins 1994), between epileptic patients taking carbamazepine or phenytoin and healthy volunteers. In accordance with the present study, the AUC and elimination $\mathrm{t} 1 / 2$ of midazolam were markedly decreased in the epileptic patients. In addition, the Cmax of midazolam was also markedly decreased in the epileptic patients. On the other hand, in the present study carbamazepine caused no significant change in the Cmax of alprazolam. The bioavailability of midazolam is low (33\%) (Olkkola et al. 1993), whereas that of alprazolam is high (92\%) (Greenblatt and Wright 1993). Therefore, it appears that midazolam, but not alprazolam, undergoes extensive firstpass metabolism. It is known that CYP3A4 is present not only in the liver but also in the small intestine (Watkins 1994). Paine et al. (1996) have reported that midazolam undergoes extensive intestinal metabolism and have suggested that this plays a major role in the firstpass metabolism of this drug. Therefore, the differential effects of carbamazepine on the Cmax of midazolam and alprazolam appear to be ascribable to the difference between the two drugs in the extent of first-pass metabolism, especially that in the small intestine.

The mean plasma alprazolam concentration at $0.5 \mathrm{~h}$ during the carbamazepine treatment was significantly higher than that during the placebo treatment. Because carbamazepine is also a substrate for CYP3A4 (Kerr et al. 1994), it may competitively inhibit the first-pass metabolism of alprazolam. However, alprazolam does not appear to undergo first-pass metabolism to a major extent as mentioned before. Therefore, we have no clear explanation for this result.

Although carbamazepine markedly decreased plasma alprazolam concentrations during the elimination phase, this was not accompanied by major changes in the psychomotor function parameters. These largely negative results may be ascribable to the sedative effect of carbamazepine itself (Albani et al. 1995), although the lack of psychomotor function data prior to carbamazepine treatment prevents us from drawing a conclusion on this problem. The significantly slower thinking speed at $1 \mathrm{~h}$ during the carbamazepine treatment may be associated with the relatively higher plasma alprazolam concentrations than during the placebo treatment, increasing plasma carbamazepine concentrations after dosing or the combination of both.

In the present study, carbamazepine caused on average a 2.4-fold increase in the CLoral of alprazolam. This result suggests that the patients taking carbmazepine require on average a 2.4 times higher dose than those not taking carbamazepine to achieve therapeutic plasma concentrations. Meanwhile, carbamazepine addition to the patients taking alprazolam appears to cause on average a $60 \%$ decrease in plasma concentrations, which may result in a clinical deterioration. In fact, Arana et al. (1988) have reported the case of a patient with atypical 
bipolar disorder and panic attacks treated with alprazolam whose clinical status markedly deteriorated after carbamazepine addition. Furthermore, carbamazepine withdrawal from the patients taking both carbamazepine and alprazolam may result in a clinical toxicity.

Finally, in the present study carbamazepine treatment with a rather short period of 10 days was used for an ethical reason. However, it should be noted that carbamazepine treatment with a longer period may further induce the metabolism of alprazolam, because the enzyme-inducing effect of carbamazepine appears to be maximal at 3-5 weeks (Bertilsson and Tomson 1986).

In conclusion, the present study suggests that carbamazepine decreases plasma concentration of alprazolam by inducing its metabolism. This drug interaction should be kept in mind when prescribing both drugs concomitantly. The present study also supports previous studies suggesting that alprazolam is metabolized predominantly by CYP3A4.

\section{ACKNOWLEDGMENT}

This study was supported by a grant from the Hirosaki Research Institute for Neurosciences.

\section{REFERENCES}

Albani F, Riva R, Baruzzi A (1995): Carbamazepine clinical pharmacology: A review. Pharmacopsychiatry 28:235244

Arana GW, Goff DC, Friedman H, Ornsteen M, Greenblatt DJ, Black B, Shader RI (1986): Does carbamazepineinduced reduction of plasma haloperidol levels worsen psychotic symptoms? Am J Psychiatry 143:650-651

Arana GW, Epstein S, Molloy M, Greenblatt DJ (1988): Carbamazepine-induced reduction of plasma alprazolam concentrations: A clinical case report. J Clin Psychiatry 49:448-449

Backman JT, Olkkola KT, Ojala M, Laaksovirta H, Neuvonen PJ (1996): Concentrations and effects of oral midazolam are greatly reduced in patients treated with carbamazepine or phenytoin. Epilepsia 37:253-257

Bertilsson L, Tomson T (1986): Clinical pharmacokinetics and pharmacological effects of carbamazepine and carbamazepine-10,11-epoxide. An update. Clin Pharmacokinet 11:177-198

Bertilsson L, A-Wistedt A, Liden A, Otani K, Spina E (1988): Alprazolam does not inhibit the metabolism of nortriptyline in depressed patients or inhibit the metabolism of desipramine in human liver microsomes. Ther Drug Monit 10:213-223

Greenblatt DJ, Shader RI, Harmatz JS, Franke K, Koch-Weser J (1977): Absorption rate, blood concentrations, and early response to oral chlordiazepoxide. Am J Psychiatr 134:559-562
Greenblatt DJ, Wright CE (1993): Clinical pharmacokinetics of alprazolam. Therapeutic implications. Clin Pharmacokinet 24:453-471

Kerr BM, Thummel KE, Wurden CJ, Klein SM, Kroets DL, Gonzalez FJ, Levy RH (1994): Human liver carbamazepine metabolism. Role of CYP3A4 and CYP2C8 in 10,11epoxide formation. Biochem Pharmacol 47:1969-1979

Lingjaerde O, Ahlfors UG, Bech P, Dencker SJ, Elgen K (1987): The UKU side effect rating scale. A new comprehensive rating scale for psychotropic drugs and a cross-sectional study of side effects in neuroleptic-treated patients. Acta Psychiatr Scand 76 (suppl 334):11-100

Nagasaki T, Ohkubo T, Sugawara K, Yasui N, Otani K, Kanetko S (1997): Determination of alprazolam and $\alpha$-hydroxyalprazolam in plasma by high-performance liquid chromatography. Anal Sci 13:245-249

Olkkola KT, Aranko K, Luurila H, Hiller A, Saarnivaara L, Himberg JJ, Neuvonen PJ (1993): A potentially hazardous interaction between erythromycin and midazolam. Clin Pharmacol Ther 53:298-305

Olkkola KT, Backman JT, Neuvonen PJ (1994): Midazolam should be avoided in patients receiving the systemic antimycotics ketoconazole or itraconazole. Clin Pharmacol Ther 55:481-485

Otani K, Yasui N, Kaneko S, Ohkubo T, Osanai T, Sugawara K (1996a): Carbamazepine augmentation therapy in three patients with trazodone-resistant unipolar depression. Int Clin Psychopharmacol 11:55-57

Otani K, Ishida M, Kaneko S, Mihara K, Ohkubo T, Osanai T, Sugawara K (1996b): Effects of carbamazepine coadministration on plasma concentrations of trazodone and its active metabolite, m-chlorophenylpiperazine. Ther Drug Monit 18:164-167

Otani K, Yasui N, Kaneko S, Ohkubo T, Osanai T, Sugawara K, Hayashi K, Chiba K, Ishizaki T (1997a): Effects of genetically determined S-mephenytoin 4-hydroxylation status and cigarette smoking on the single-dose pharmacokinetics of oral alprazolam. Neuropsychopharmacology 16:8-14

Otani K, Ishida M, Yasui N, Kondo T, Mihara K, Suzuki A, Furukori H, Kaneko S, Inoue Y (1997b): Interaction between carbamazepine and bromperidol. Eur J Clin Pharmacol 52:219-222

Paine MF, Shen DD, Kunze KL, Perkins JD, Marsh CL, McVicar JP, Barr DM, Gillies BS, Thummel KE (1996): First-pass metabolism of midazolam by the human intestine. Clin Pharmacol Ther 60:14-24

Pirmohamed M, Allott R, Green VJ, Kitteringham NR, Chadwick D, Park BK (1994): Lymphocyte microsomal epoxide hydrolase in patients on carbamazepine therapy. Br J Clin Pharmacol 37:577-581

Post RM, Ketter TA, Pazzaglia PJ, Denicoff K, Marangell L, George MS (1994): Anticonvulsants in refractory mood disorders. In Nolen WA, Zohar J, Roose SP, Amsterdam JD (eds), Refractory Depression: Current Strategies and Future Directions. Chichester, U.K., John Wiley, pp 97114

Siris SG (1993): Adjunctive medication in the maintenance treatment of schizophrenia and its conceptual implications. Br J Psychiatry 163:66-78 
von Moltke LL, Greenblatt DJ, Cotreau-Bibbo MM, Harmatz JS, Shader R (1994): Inhibitors of alprazolam metabolism in vitro: Effect of serotonin-reuptake-inhibitor antidepressants, ketokonazole and quinidine. Br J Clin Pharmacol 38:23-31

Watkins PB (1994): Noninvasive tests of CYP3A enzymes. Pharmacogenetics 4:171-184

Wietholtz H, Zysset T, Kreiten K, Kohl D, Buchsel R, Matern $S$ (1989): Effect of phenytoin, carbamazepine, and val- proic acid on caffeine metabolism. Eur J Clin Pharmacol 36:401-406

Yasui N, Otani K, Kaneko S, Ohkubo T, Osanai T, Sugawara K, Chiba K, Ishizaki T (1996): A kinetic and dynamic study of oral alprazolam with and without erythromycin in humans: In vivo evidence for the involvement of CYP3A4 in alprazolam metabolism. Clin Pharmacol Ther 59:514-519

Yue QY, Tomson T, Sawe J (1994): Carbamazepine and cigarette smoking induce differentially the metabolism of codeine in man. Pharmacogenetics 4:193-198 